ETH-TH/99-15

\title{
Two photons plus jet at LHC: the NNLO contribution from the $g g$ initiated process ( $^{2}$
}

\author{
D. de Florian and Z. Kunszt \\ Institute of Theoretical Physics, ETH \\ CH-8093 Zürich, Switzerland
}

\begin{abstract}
The production of the Standard Model Higgs boson of mass $\approx 100-140 \mathrm{GeV}$ at the LHC likely gives clear signals in the $\gamma \gamma(1)$ and in the $\gamma \gamma$ jet (2) channels. The quantitative evaluation of the background to channel (1) is very hard since the nextto-leading order (NLO) and next-to-next-to-leading order (NNLO) QCD corrections are large. In particular, the contributions of the NNLO QCD subprocess $g g \rightarrow \gamma \gamma$ to inclusive $\gamma \gamma$ production is comparable to the contribution of the leading order subprocess $q \bar{q} \rightarrow \gamma \gamma$. The quantitative description of the background to channel (1), therefore, requires to calculate all corrections up to the NNNLO level. In this letter we present results on the contribution of the NNLO QCD subprocess $g g \rightarrow g \gamma \gamma$ to the production rate of channel (2). We have found that in this case this NNLO contribution is less than $20 \%$ of the Born contribution. Since the NNLO contributions will likely be dominated by this subprocess one can argue that in the case of channel (2) - contrary to the case of channel (1) - a quantitative description of the background can be achieved already at next-to-leading order accuracy.
\end{abstract}

\footnotetext{
${ }^{1}$ Work partly supported by the EU Fourth Framework Programme 'Training and Mobility of Researchers', Network 'Quantum Chromodynamics and the Deep Structure of Elementary Particles', contract FMRX-CT98-0194 (DG 12 - MIHT).
} 
The search for Standard Model Higgs boson will be a high priority project at the LHC collider. Recent results of the LEP experiments obtained from a fit to the precision data and by direct search indicate that the mass of the Higgs boson at the $95 \%$ confidence level should be in the mass range of $90 \mathrm{GeV} \leq m_{H} \leq 262 \mathrm{GeV}$ [1]. However, the search for the Higgs boson at the LHC is rather difficult in the low mass range of $100 \mathrm{GeV} \leq m_{H} \leq 140$ $\mathrm{GeV}$ when measurable signals exist for the two photon decay channel. The simulations carried out by ATLAS and CMS [2] have shown that the significance of the signal of Higgs production in the inclusive channel $p p \rightarrow \gamma \gamma$ is rather low. Assuming integrated luminosity of $3 \cdot 10^{-4} \mathrm{pb}^{-1}$ one obtains $S / \sqrt{B} \approx 5-10$, the actual value depending on the Higgs mass and the assumptions on the size of the higher order QCD corrections.

Next-to-leading order corrections to the signal are known to be large [3] (with a $K$ factor of $\approx 1.8$ ), whereas the full calculation of the background, including fragmentation effects, at the same order is just being evaluated 四. The NLO results, however, will likely be not enough for having a quantitative description of the background. At LO only the $q \bar{q} \rightarrow \gamma \gamma$ subprocess can contribute and, for this initial state, the parton-luminosity is rather low. It is not surprising, therefore, that the one-loop subprocess $g g \rightarrow \gamma \gamma$ (box diagram) [5], appearing first in NNLO accuracy but having high initial parton-luminosity and collinear singularity structure similar to a Born one, gives production-rates which are numerically very similar (both in size and shape) to the LO result. This poses a serious theoretical problem, since in order to achieve a significant reduction in the scale dependence one has to go one order further, that is to NNNLO, which is completely beyond the scope of the available techniques.

Recently, it has been pointed out [6] that the production of the Higgs boson in association with a hard jet, and its subsequent decay to a photon pair, also gives measurable signal. This channel has both experimental and theoretical advantages. The detection of the more energetic photons in association with a hard jet allows to improve the efficiency and the two photon invariant mass resolution. Furthermore, the existence of a jet in the final state allows for the implementation of different cuts which can be particularly useful to suppress the background. The cross-section of the signal process $p p \rightarrow H$ jet has recently 
been computed to NLO accuracy, in the limit of $m_{H} \leq m_{t}$, finding a $K$-factor similar to the more inclusive case $K \sim 1.6$ [7]. Although all the NLO matrix elements needed to construct the corresponding NLO Monte Carlo program for numerical evaluation of the background are available [8], such a program has not been implemented yet. Furthermore, the theoretical problem of the gluon-gluon initial state pointed out for the inclusive channel may also be an issue in this case. In the Born approximation, the background receives contributions from the subprocesses $q \bar{q} \rightarrow g \gamma \gamma$ and $q g \rightarrow q \gamma \gamma$. The subprocess with gluon-gluon initial state $g g \rightarrow g \gamma \gamma$ (box and pentagon diagrams) contributes again first in NNLO accuracy. But there is an important difference: in this case, subprocesses with gluon-quark initial state having a high parton-luminosity contribute already at the lowest order. It is, therefore, expected that the NNLO $g g$ contribution will be far less significant than in the inclusive case. If this expectation turns out to be correct, then one may argue that a quantitative description of the background can be achieved already at next-to-leading order accuracy. The evaluation of this $g g$ initiated NNLO correction for the production of two photons plus one jet at the LHC is the main purpose of this letter.

The one loop $3 g 2 \gamma$ amplitudes have not been directly computed. But fortunately, the one loop results of Bern, Dixon and Kosower [9] on the $5 g$ amplitudes have been documented in terms of partial amplitudes with explicit color decomposition; therefore one can obtain the $3 g 2 \gamma$ amplitudes by changing color factors, normalizations and doing appropriate permutations 2 .

The $n_{f}$ part of the one loop $5 g$ amplitude can be decomposed in the following way

$$
\mathcal{A}_{5\left[n_{f}\right]}^{1-\text { loop }}=g^{5} \sum_{\sigma \in S_{5} / Z_{5}} \operatorname{Tr}\left[T^{a_{\sigma(1)}} T^{a_{\sigma(2)}} T^{a_{\sigma(3)}} T^{a_{\sigma(4)}} T^{a_{\sigma(5)}}\right] A_{5 ; 1}^{[1 / 2]}(\sigma(1), \sigma(2), \sigma(3), \sigma(4), \sigma(5))
$$

where $S_{5} / Z_{5}$ is the set of the 24 non-cyclic permutations of five objects and $A_{5 ; 1}^{[1 / 2]}$ is the partial amplitude corresponding to the case of a fermion circulating in the loop. The $T^{a}$ are the SU(3) generators in the fundamental representation, normalized so that $\operatorname{Tr}\left(T^{a} T^{b}\right)=\delta^{a b}$.

The one loop $3 g 2 \gamma$ amplitude (with the photons labeled by 4 and 5 ) has the same color

\footnotetext{
${ }^{2}$ We thank L. Dixon for his assistance concerning these modifications.
} 
decomposition and it can be obtained [8] by replacing in eq.(1) the corresponding $\mathrm{SU}(3)$ generators of the gluons by the $\mathrm{U}(1)$ values (for each photon) and the QCD coupling $g$ by the electric charge of the quark circulating within the loop, as

$$
\begin{gathered}
T^{a_{4}} \rightarrow \sqrt{2} \\
T^{a_{5}} \rightarrow \sqrt{2} \\
g^{5} \rightarrow g^{3} e_{Q}^{2} .
\end{gathered}
$$

In the color decomposition for the 3 remaining gluons, after these replacements, only two color structures appear, i.e

$$
\begin{aligned}
\mathcal{A}_{3 g 2 \gamma}^{1-\text { loop }}(1,2,3 ; 4,5)= & g^{3}\left(e_{Q} \sqrt{2}\right)^{2} \\
& \left\{\operatorname{Tr}\left[T^{a_{1}} T^{a_{2}} T^{a_{3}}\right] A_{5}^{2 \gamma}(1,2,3 ; 4,5)+\operatorname{Tr}\left[T^{a_{3}} T^{a_{2}} T^{a_{1}}\right] A_{5}^{2 \gamma}(3,2,1 ; 4,5)\right\}
\end{aligned}
$$

where $A_{5}^{2 \gamma}(1,2,3 ; 4,5)$ is obtained by collecting the 12 permutations of $A_{5 ; 1}^{[1 / 2]}$ with the right cyclic ordering of $(1,2,3)$,

$$
\begin{aligned}
A_{5}^{2 \gamma}(1,2,3 ; 4,5) & =A_{5 ; 1}^{[1 / 2]}(1,2,3,4,5)+A_{5 ; 1}^{[1 / 2]}(1,2,3,5,4)+A_{5 ; 1}^{[1 / 2]}(1,2,4,3,5) \\
& +A_{5 ; 1}^{[1 / 2]}(1,2,5,3,4)+A_{5 ; 1}^{[1 / 2]}(1,2,4,5,3)+A_{5 ; 1}^{[1 / 2]}(1,2,5,4,3) \\
& +A_{5 ; 1}^{[1 / 2]}(1,4,2,3,5)+A_{5 ; 1}^{[1 / 2]}(1,4,2,5,3)+A_{5 ; 1}^{[1 / 2]}(1,4,5,2,3) \\
& +A_{5 ; 1}^{[1 / 2]}(1,5,2,3,4)+A_{5 ; 1}^{[1 / 2]}(1,5,2,4,3)+A_{5 ; 1}^{[1 / 2]}(1,5,4,2,3) .
\end{aligned}
$$

Furthermore, by using Furry's theorem one obtains

$$
A_{5}^{2 \gamma}(1,2,3 ; 4,5)=-A_{5}^{2 \gamma}(3,2,1 ; 4,5)
$$

The partial amplitudes $A_{5 ; 1}^{[1 / 2]}$ for the four independent helicity structures $(++++$ $+),(-++++),(--+++),(-+-++)$ are given in ref. [9]. The remaining ones are obtained by cyclic permutations or complex conjugation. The same method can be used to re-compute the $2 g 2 \gamma$ amplitudes from the $4 g$ ones evaluated in ref. [10]. We have verified numerically soft and collinear limits for the $A_{5}^{2 \gamma}$ helicity amplitudes as well as for their spin averaged squared values. 
The cross-section values presented below have been obtained using the LO MRST [11] parton distributions and with the factorization and renormalization scales given by

$$
Q_{0}^{2}=M_{\gamma \gamma}^{2}+p_{T(j e t)}^{2},
$$

where $M_{\gamma \gamma}$ corresponds to the invariant mass of the photon pair. The jet and the photons are required to have $p_{T}>40 \mathrm{GeV}$ and rapidity within $|\eta|<2.5$, i.e., corresponding to the $\mathbf{C} \mathbf{1}$ cuts introduced in the analysis of ref. [6]. Furthermore, since photons should be isolated, we apply a cut $\Delta R=\sqrt{\Delta \phi^{2}+\Delta \eta^{2}}>0.3$ for each pair of particles, avoiding in this way any possible $q \gamma$ final state collinear singularity. In this work, we are only interested in the direct production of two photons, so we neglect any contribution coming from the single and double fragmentation processes. Since the $\gamma \gamma$ measurement is intended for a detection of a Higgs boson lighter than the top quark, we assume only 5 flavors and consider all of them massless. This has been shown [5] to be a very good approximation for the box contribution, where the massive expressions are also known 12 .

In order to make comparison with the case of the inclusive channel $p p \rightarrow \gamma \gamma$ we have also evaluated the corresponding $q \bar{q} \rightarrow \gamma \gamma$ and $g g \rightarrow \gamma \gamma$ contributions with similar cuts for the photons $\left(p_{T}>40 \mathrm{GeV},|\eta|<2.5\right)$ and $Q_{0}^{2}=M_{\gamma \gamma}^{2}$ as default scale.

In Fig. 1(a) we show the results for the inclusive channel, from where one can see that the NNLO $(g g)$ contribution is rather large and actually it is comparable to the lowest order $(q \bar{q})$ one, as stated before. In Fig. 1(b) we show the lowest order $(q g$ and $q \bar{q})$ and NNLO $(g g)$ contributions for the case of $\gamma \gamma$ jet production. It is clear from the plot that, as in the inclusive case, the NNLO $g g$ initiated contribution is similar both in magnitude and shape to the $q \bar{q}$ initiated one. But since the Born cross section is dominated by the $q g$ subprocess, the $g g$ NNLO term becomes less significant than for the inclusive channel. Of course, since all these contributions for each partonic initial state are 'Born-like' ones, the results presented in Fig. 1 are strongly scale dependent. In order to study the effect of using different scales, in Fig. 2 we show the scale dependence of the ratio between the NNLO $g g$ initiated and the Born contributions $R=\sigma^{g g} / \sigma^{L O}$, for both the inclusive and the jet channel and for 3 different values of the invariant mass of the photon pair $M_{\gamma \gamma}=100,120,140 \mathrm{GeV}$. We vary the default scales by introducing a parameter $\mu$ such 

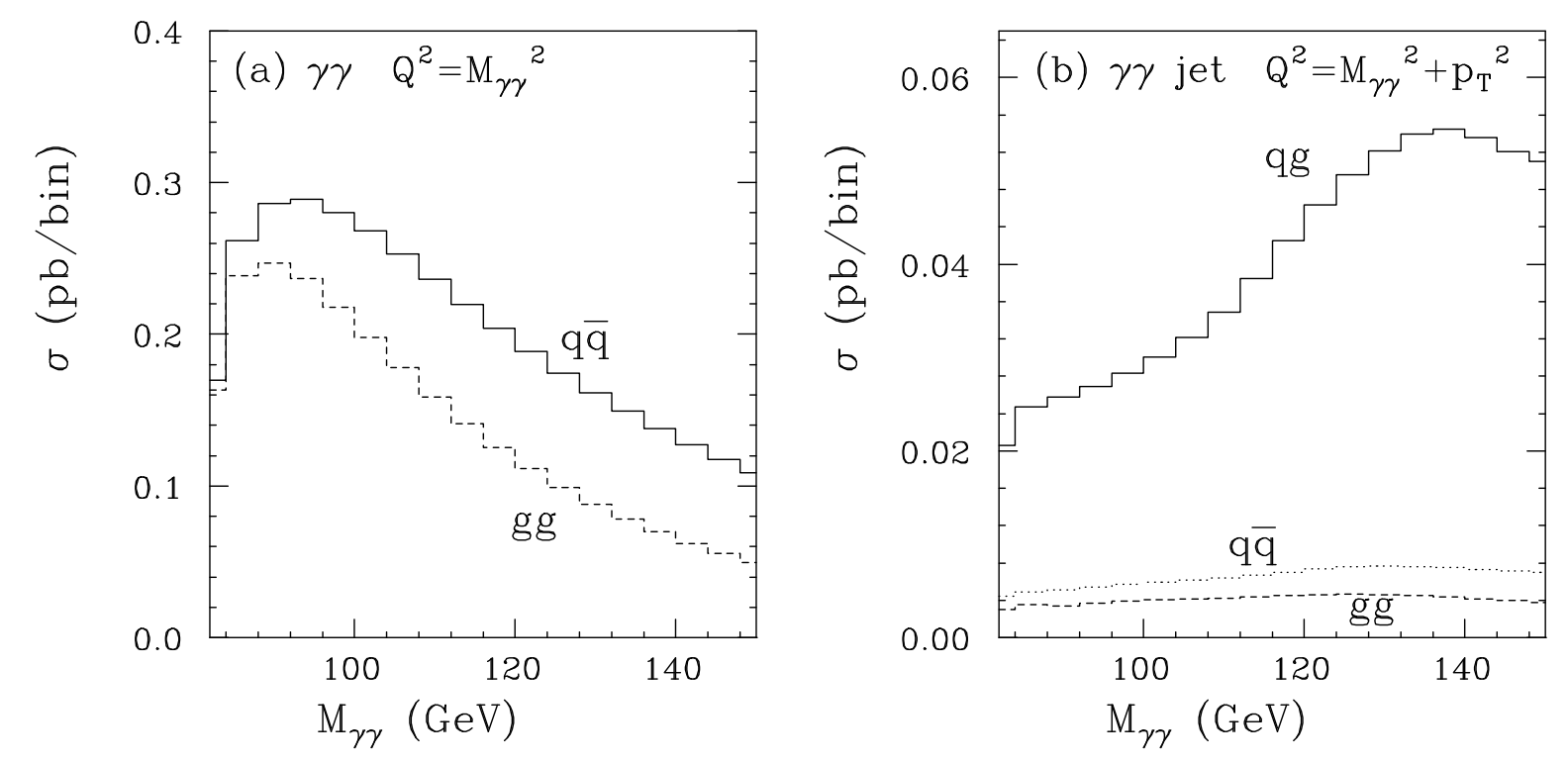

Figure 1: Contributions of the different partonic initial states to the cross-section for processes (a) $p p \rightarrow \gamma \gamma$ and (b) $p p \rightarrow \gamma \gamma$ jet.

that $Q=\mu Q_{0}$, where $Q_{0}$ corresponds to the default scale defined above for each process. As it can be observed from Fig. 2, even though the scale dependence for the ratios are quite similar, in the jet channel the $g g$ contribution remains always smaller than $20 \%$ of the Born one, whereas for the inclusive channel it can even be larger than the LO term at the lower scales.

In conclusion, we have presented results on the contribution of the NNLO QCD subprocess $3 g 2 \gamma$ to the production-rate of two photons plus one jet at the LHC. We have found that this NNLO contribution is less than $20 \%$ of the Born contribution. Since the NNLO contributions will likely be dominated by this subprocess, one can argue that in the case of associated production of a Higgs boson with one jet - contrary to the case of the completely inclusive production - one can achieve a quantitative description of the background already in next-to-leading order accuracy. This provides us another argument for favoring the $\gamma \gamma$ jet signal in searching for the Standard Model Higgs boson in the mass range of $m_{H} \approx 100-140 \mathrm{GeV}$. 

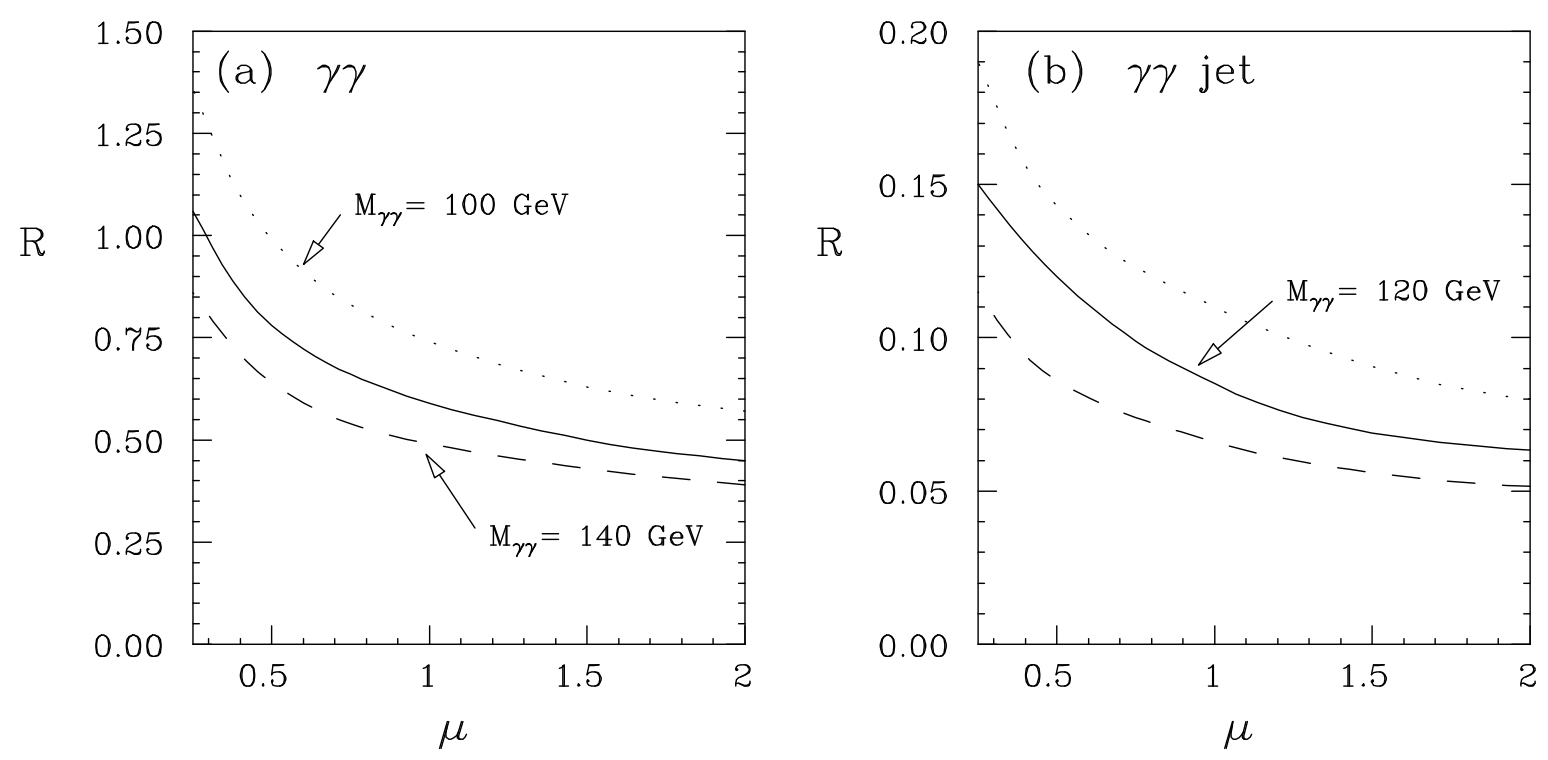

Figure 2: Ratio of the NNLO $g g$ initiated contribution over the LO one for (a) $p p \rightarrow \gamma \gamma$ and (b) $p p \rightarrow \gamma \gamma$ jet.

We are grateful to L. Dixon, M. Grazzini and A. Signer for helpful discussions.

\section{References}

[1] LEP Collaborations and Electroweak Working Group, CERN-EP/99-15 (1999).

[2] ATLAS Collaboration, Technical Proposal, CERN/LHCC 94-93 (1994);

CMS Collaboration, Technical Design Report, CERN/LHCC 97-33 (1997) 27;

C. Seez et al., Proc. Large Hadron Collider Workshop, Aachen, CERN-90-10-

B/ECFA 90-133, Vol.II, p.474 (1990);

D. Froidevaux, F. Gianotti and E. Richter-Was, ATLAS Internal Note, Phys-No-64 (1995).

[3] D. Graudenz, M. Spira and P. Zerwas, Phys. Rev. Lett. 70, 1372 (1993);

M. Spira, A. Djouadi, D. Graudenz and P. Zerwas, Nucl. Phys. B 453, 17 (1995).

[4] J. P. Guillet, presentation at the LHC Workshop, CERN, Jan. 1999. 
[5] D. Dicus and S. Willenbrock, Phys. Rev. D 37, 1801 (1988).

[6] S. Abdullin, M. Dubinin, V. Ilyin, D. Kovalenko, V. Savrin and N. Stepanov, Phys. Lett. B 431, 410 (1998).

[7] D. de Florian, M. Grazzini and Z. Kunszt, hep-ph/9902483 (1999).

[8] Z. Bern, L. Dixon and D. Kosower, Nucl. Phys. B437, 259 (1995);

A. Signer, Phys. Lett. B 357, 204 (1995).

[9] Z. Bern, L. Dixon and D. Kosower, Phys. Rev. Lett. 70, 2677 (1993).

[10] Z. Kunszt, A. Signer and Z. Trócsányi, Nucl. Phys. B411, 397 (1994).

[11] A.D. Martin, R.G. Roberts, W.J. Stirling and R.S. Thorne, Eur. Phys. J C4, 463 (1998).

[12] V. Constantini, B. De Tollis and G. Pistoni, Nuovo Cimento 2A, 733 (1971). 